\title{
Effect of Alcohol Consumption on Oxidative Stress Markers and its Role in the Pathogenesis and Progression of Liver Cirrhosis
}

\author{
Neelesh Deshpande ${ }^{1}$, Sabitha Kandi², P Venkata Bharath Kumar ${ }^{3}$, K V Ramana ${ }^{4, *}$, Manohar Muddeshwar ${ }^{1}$ \\ ${ }^{1}$ Department of Biochemistry, Government Medical college, Nagpur \\ ${ }^{2}$ Department of Biochemistry, Chalmeda Anandarao Inastitute of Medical Sciences, Karimnagar \\ ${ }^{3}$ Department of Biochemistry, Vaydehi Institute of Medical Sciences and research center, Bengaluru \\ ${ }^{4}$ Department of Microbiology, Prathima Institute of Medical Sciences, Karimnagar \\ *Corresponding author: ramana_20021@rediffmail.com
}

Received September 02, 2013; Revised October 10, 2013; Accepted October 11, 2013

\begin{abstract}
Oxidative stress has been increasingly implicated in the pathogenesis and progression of liver cirrhosis. Chronic ethanol consumption induces an oxidative stress resulting in increased ferritin levels and thereby iron over load. The study was aimed at evaluating the relation between alcohol consumption and erythrocyte superoxide dismutase (SOD), Glutathione peroxidase (GPx), Malondialdehyde (MDA) activities in liver cirrhosis patients. The study included two groups based on alcohol consumption; subjects taking low alcohol content (Less than $150 \mathrm{~g} /$ day.), those consuming high alcohol content (More than $150 \mathrm{~g} /$ day), diagnosed as suffering from liver cirrhosis and control group who do not take alcohol and are not suffering from liver cirrhosis. All cirrhotic patients on high alcohol intake in the study group had lower serum SOD ( $<0.0001)$, GPx $(0.0001)$ and significantly higher MDA levels $(\mathrm{P}<0.001)$ than those with subjects taking low alcohol and control group. These results suggest that the decrease in erythrocyte SOD, GPx and increase in MDA levels are related to the alcohol consumption and that may be associated with pathogenesis and progression of liver disease.
\end{abstract}

Keywords: liver cirrhosis, alcohol consumption, malondialdehyde, superoxide dismutase, Glutathione peroxidase

Cite This Article: Neelesh Deshpande, Sabitha Kandi, P Venkata Bharath Kumar, K V Ramana, and Manohar Muddeshwar, "Effect of Alcohol Consumption on Oxidative Stress Markers and its Role in the Pathogenesis and Progression of Liver Cirrhosis." American Journal of Medical and Biological Research 1, no. 4 (2013): 99-102. doi: 10.12691/ajmbr-1-4-3.

\section{Introduction}

Oxidative stress is defined as an imbalance between pro-oxidant and antioxidant mechanisms in human where the balance is being tilted in favor of the former [1]. Ethanol plays a significant role in the pathogenesis and progression of liver disease. Though liver breaks down the majority of ingested ethanol, it cannot metabolize beyond a specific threshold level [2]. Oxidative stress plays an important role both in non-infectious and infectious conditions such as anemia, hepatocellular carcinomas and infectious diseases $[3,4,5,6]$. The available literature suggests that intermediates of oxygen reduction may be associated with the development and progression of liver diseases [7].

Increased production and accumulation of free radical in liver plays an important role in the pathogenesis and progression of liver disease $[8,9,10,11]$. Previous studies have confirmed that a lipid peroxidation product, malondialdehyde(MDA), stimulates directly or indirectly the lipocytes (Ito cells) resulting in liver fibrosis $[12,13,14]$. Existing literature has established the fact that alcohol consumption induces lipid peroxidation and oxidative stress [15]. Peroxidative lipid decomposition is suggested as a mechanism of hepatocellular injury in parenchymal iron overload [16]. These findings point out the importance of iron and MDA in the progression of liver cirrhosis (LC) [17]. Moreover, little is known about the role of Superoxide dismutase (SOD) in LC. The aim of this study is to evaluate activities of superoxide dismutase (SOD), glutathione peroxidase (GPx) and malondialdehyde (MDA), in chronic alcoholic and cirrhotic patients (low and high alcohol consumption) and to determine the effect of increasing alcohol intake on the pathogenesis and progression of liver disease.

\section{Patients and Methods}

The study included fifty patients with alcoholic liver cirrhosis and the study subjects are divided in to two groups based on alcohol consumption. Subjects included were grouped as those consuming low alcohol content (less than $150 \mathrm{~g} /$ day.) (ALC1), consuming high alcohol content (more than $150 \mathrm{~g} /$ day) (ALC2) and control group who do not consume alcohol and are not suffering from 
liver cirrhosis. 28 patients were included in low alcohol consuming group (ALC1) with a mean age of $42 \pm 8$ years and 22 patients were included in high alcohol consuming group (ALC2) with a mean age of $42 \pm 8$ years. 90 individuals with no history of alcohol consumption and without liver cirrhosis are recruited as controls in the study. Patients were obtained from gastroenterology ward of Government medical college and superspeciality teaching hospital with proven history of liver cirrhosis on the basis of clinical, biochemical and imaging methods and endoscopic signs. The severity of the disease was evaluated according to the Child-Pugh classification [18]. Informed and written consent was obtained from each patient included and the study and was approved by institutional ethical committee.

\subsection{Exclusion Criteria}

Patients with non alcoholic cirrhosis due to hepatitis B, hepatitis C, wilsons disease, haemochromatosis, $\alpha_{1}$ antitrypsin deficiency, toxic damage to the liver and having history of vitamin supplementation or antibiotic therapy were excluded. Blood samples were drawn from patients with LC on first day of admission.

\subsection{Markers of Liver Injury and Oxidative Stress}

Serum total bilirubin, Serum aspartate transaminase (AST), albumin, and gamma glutamyl transferase (GGT) were measured in all of the patients. SOD, GPx, MDA levels were measured in 50 patients with cirrhosis and the results obtained were correlated with normal individual's age matched and having no history of LC.

\subsection{SOD Measurement (U/gm/Hb)}

The Quantitative in vitro determination of Superoxide Dismutase was performed by Randox Laboratories method. Erythrocytes were obtained for the superoxide dismutase activity which was measured using xanthine and xanthine oxidase (XOD) to generate superoxide radicals which react with 2- (4-iodophenyl)-3-(4nitrophenol)-5-phenyltetrazolium chloride (I.N.T) to form a red formazan dye. The superoxide activity is then measured by the degree of inhibition of this reaction. One unit of SOD is that which causes $50 \%$ inhibition of the rate of reduction of INT under the conditions of the assay. The absorbance was measured at $505 \mathrm{~nm}$ [19].

\subsection{Glutathione Measurement (U/gm/Hb)}

The Quantitative in vitro determination of glutathione Peroxidase (GPx) was performed from Randox Kit. The assay method utilizes glutathione oxidation by cumine hydroxide. The oxidation of NADPH to $\mathrm{NADP}^{+}$was measured by the decrease in absorbance at $340 \mathrm{~nm}$ [20].

\subsection{Lipid Per-oxidation Measurement (MDA (nmol/ml))}

The MDA was estimated by the standard method where the Serum lipid peroxide was mixed with Methyl Phenyl indole in acidified medium and after incubation for $48^{\circ} \mathrm{C}$ for 60 minutes, the resultant chromophore intensity was recorded at $586 \mathrm{~nm}$ [21].

\subsection{Ferritin Estimation}

Ferritin was estimated on the VIDAS (VITEK Immuno Diagnostic Assay System) instruments. VIDAS Ferritin is an automated quantitative test for use on the VIDAS instruments for the determination of human Ferritin in human serum using enzyme linked fluorescent assay (ELFA) technique. The assay combines a one-step immunoassay sandwich method with a final fluorescent detection. During the detection step, the substrate (4Methyl umbelliferyl phosphate) is cycled in and out of the Solid Phase Receptacle (SPR). The conjugate enzyme catalyses the hydrolysis of this substrate in to a florescent product (4-Methyl umbelliferone) and the resulting florescence is measured at $450 \mathrm{~nm}$. The intensity of the florescent is proportional to the concentration of antigens present in the sample [22,23,24].

\subsection{Statistical Analysis}

The continuous variables were recorded as a Mean \pm standard deviation. Statistical analysis was performed with open-epi software. The significance of difference between the various groups was calculated using the Correlation, ANOVA-f test to ascertain whether the differences between the various groups were clinically significant $(P$ $<0.001)$.

Table 1. Oxidative stress markers in controls and cirrhotic patients

\begin{tabular}{|c|c|c|c|}
\hline \multirow{2}{*}{ Variables } & $\begin{array}{c}\text { CONTROLS } \\
\mathrm{n}=90\end{array}$ & $\begin{array}{c}\text { Case Group 1 (ALC1) } \\
\mathrm{N}=28\end{array}$ & $\begin{array}{c}\text { Case Group 2 (ALC2) } \\
\mathrm{N}=22\end{array}$ \\
\hline MDA nmol/ml & $3.03 \pm 0.96$ & $9.23 \pm 1.29$ & $10.76 \pm 1.03$ \\
\hline SOD & $6.97 \pm 1.69$ & $4.00 \pm 0.62$ & $3.26 \pm 0.64$ \\
\hline GPX & $13.04 \pm 1.74$ & $9.28 \pm 1.39$ & $8.38 \pm 1.05$ \\
\hline Ferritin & $68.85 \pm 19.39$ & $<0.0001$ & $<.001$ \\
\hline
\end{tabular}

ALC1 - Alcohol consumption less than 150 g/day.

ALC2- Alcohol Exposure more than 150 g/day.

MDA-Malondialdehyde

SOD- superoxide dismutase

GPX- Glutathione peroxidase 
Table 2. Serum AST, T.Bilirubin, Albumin, GTT levels of patients

\begin{tabular}{|c|c|c|c|}
\hline LIVER FUNCTION TESTS & $\begin{array}{c}\text { CONTROLS } \\
\mathrm{n}=90 \\
\text { Mean } \pm \text { SD }\end{array}$ & $\begin{array}{c}\text { Case Group 1 (ALC1) } \\
\mathrm{n}=28\end{array}$ & $\begin{array}{c}\text { Case Group 2 (ALC2) } \\
\mathrm{n}=22\end{array}$ \\
\hline AST U/ml value & $130.29 \pm 43.92$ & $6.20 \pm 2.78$ \\
\hline T.BILURUBIN mg/dl & $34.04 \pm 6.88$ & $5.77 \pm 3.51$ & $<0.0001$ \\
\hline ALBUMIN g/dl & $0.62 \pm 0.16$ & $2.82 \pm 0.19$ & $2.51 \pm 0.33$ \\
\hline GGT U/ml & $3.65 \pm 0.23$ & $<0.0001$ & $<2.36$ \\
\hline
\end{tabular}

ALC1 -Alcohol consumption less than 150 g/day.

ALC2- Alcohol Exposure more than 150 g/day.

GGT- gamma-glutamyl transpeptidase

AST-Alaninie Aspartate transaminase

\section{Results}

The differences between the two groups of alcoholic cirrhotic patients and control subjects in terms of liver injury (GGT, AST, albumin and T.bilurubin) were statistically significant. All cirrhotic patients showed significant decrease in SOD ( $p<0.0001)$ and GPx (p < 0.0001 )and corresponding increase in MDA levels ( $\mathrm{p}<$ $0.001)$ and ferritin levels $(\mathrm{p}<0.0001)$ when compared with control subjects as shown in Table 1. Serum AST levels and total bilirubin levels were found to be higher in cirrhotic patients with ALC2 than those with ALC1. Serum albumin levels were found to be lower in cirrhotic patients with ALC2 than those with ALC1. Serum GTT levels were found to be lower in patients with ALC2 than in ALC1 as shown in Table 2. Correlation coefficient (PEARSON'S) between the oxidative stress markers with indicators of liver injury in different alcohol consumption groups ( $r=-0.072$ for ALC1 and $r=-0.126$ for ALC2) in liver cirrhosis was weakly significant.

\section{Discussion}

The study results have indicated that there was accumulation of oxidative stress markers with increasing alcohol intake resulting in further damage to liver as revealed by abnormal liver function tests. This study results were similar to previous research reports which suggested that there was an increased risk of liver injury with chronic alcohol intake [25,26]. Studies in the past have also revealed that there was an increase in iron load with chronic ethanol exposure [16,27]. It has been experimentally demonstrated in chronic ethanol fed rats that ethanol tends to increase lipid peroxidation with iron acting as a cofactor in catalyzing lipid peroxidation and is responsible for elevated malondialdehyde (MDA) production [28].

Our study revealed that there was an increase in ferritin levels and subsequent rise in MDA levels associated with high alcohol consumption as observed by a previous study [29]. A study by Clot P et al, which assessed the role of alcohol in oxidative damage of liver, revealed that decreased activities of glutathione were observed among cirrhotic patients (alcohol consuming group) with worsening Child Pugh score when compared with control subjects confirming the fact that there was stimulation of lipid peroxidation in patients with liver cirrhosis which is associated with alcohol abuse [29].

Previous studies have demonstrated that there was an association between reduced antioxidant levels and increased oxidative stress markers with increasing alcoholic consumption resulting in liver injury [29,30,31].

The current study results have also indicated that there was an added risk of liver injury with increased alcohol consumption as evident from the abnormal liver function tests. There was as a negative correlation of oxidative stress markers with levels of alcohol consumption and markers of liver injury.

Drawback of this study is that we could not evaluate extracellular MnSOD and cytosolic CuZnSOD activities which are released from damaged cells and are indicators of cell death.

In conclusion, it is evident from the results of this study and the existing literature that there was a compromise of antioxidant defense system with rise in oxidative stress markers and subsequent decrease in antioxidants levels between the two groups with alcohol consumption when compared to normal controls. The level of oxidative stress is indicated with elevated MDA levels and corresponding reduction in SOD and GPX levels which revealed significantly increased risk of liver injury related to excessive alcohol intake when compared with control group. Our results reemphasize the fact that with diminishing antioxidant levels and the rising oxidative stress with iron as central cofactor in producing injury, alcohol consumption and iron levels play a key role in the progression of liver disease.

Regular monitoring of oxidative stress markers and iron overload indicators in alcoholic patients is necessary for better patient management and to minimize the morbidity and mortality related to liver injury.

\section{References}

[1] Halliwell B., Gutteridge JMC., Cross CE. Free radicals, antioxidants, and human disease;Where are we now? J lab Clin Med. 1992; 598-620.

[2] Savolainen, V. T., Liesto, K., Männikkö, A., Penttilä, A. and Karhunen, P. J., Alcohol Consumption and Alcoholic Liver Disease: Evidence of a Threshold Level of Effects of Ethanol. Alcoholism: Clinical and Experimental Research. 1993;17: 11121117.

[3] Abou-Seif, M.Rabia, A. and Nasr Anti oxidant status. Erythrocyte membrane lipid peroxidation and osmotic fragility in malignant lymphoma patients. Clin. Chem. Lab Med. 2000; 38:737-42. 
[4] Khanzode S.S., Muddeshwar M.G., Khanzode S.D., and Dakhale G.N. 2004, Antioxidant enzymes and lipid peroxidation in different stages of breast cancer. Free Radical Res.2004; 38(1):8185

[5] Liu, M.J., Auerbach,A.D., Anderson, S.M.,Gren, S.W. and Young, N.S. 1993. A trail of recombinant human superoxide dismutase in patients with Fanconi anaemia. Br. J. Hem., 85:406-8.

[6] Sharma P., Mishra M., Ajmera P., and Mathur S. Oxidative stress in metabolic syndrome. Indian Journal of Clinical Biochemistry. 2005; 20(1), 145-149.

[7] Agnieszka Szuster-ciesielska, Danlluk,J., Kandefer-Szerszen M. (2002) Oxidative stress in the blood of patients with alcoholrelated liver.Med Sci Monit. 2002;8(6):CR419-424.

[8] Dey A., Cederbaum A.I. Alcohol and oxidative liver Injury. 2006;43: S63-S74.

[9] Ljubuncic, T., Tane, Z and Bomzon, A. 2000. Evidence of a systemic phenomenon for oxidative stress in cholestatic liver disease. Gut. 2000; 47:710-6.

[10] Nalini, G., Hariprasad, C. and Naryanan, V.A.. Oxidative stress in alcoholic liver diseases. Indian.J. Med.Res.1999; 110:200-3.

[11] Togashi, H., Shinzowa, H., Wakabayaski, H., Nakamura, T., Yamada, V., Takahashi, T. and Ishikawa, M.. Activities of free oxygen radical scavenger enzymes in human liver. J. Hepatol., 1999; 11:200-5.

[12] Friedman, S.L. 1999. The cellular basis of hepatic fibrosis Mechanism and treatment strategies. N. Engl.J.Med.. 1999,328:1828-35.

[13] Svegliati-Baroni, G., Di Sario, A., Casini,A., Ferretti, G., D’ Ambrosio,L., Ridolfi, F.,Bolognini, L., Salzano,R., Orlandi,F.and Benedetti, A. The $\mathrm{Na}^{+} / \mathrm{H}^{+}$Exchanger modulates the Fibrogenic effect of oxidative stress in rat hepatic stelleate cells. J.Hepatol. 1999; 30:868-75.

[14] Sukamato, H.C., Kim, W., Louz, Z., Horn, W. and sul, C. Role of lipid peroxidation in vitro and in vivo models of liver fibrogenenesis. Gastroenterology.1993, 104:1012.

[15] Situnayake,RD., Crump,BJ., Thurnham,DI., Davies,JA., Gearty, J., Davis M. Lipid peroxidation and hepatic antioxidants in alcoholic liver disease. Gut, 1990, 31:1311-1317.

[16] Bruce BR., Anthony ST, Gary MB., Park CH and Richard OR.Hepatic lipid peroxidation in vivo in rats with Chronic iron overload. J. Clin. Invest.. 1983,17:429-439.

[17] Subir Kumar Das and D. M. Vasudevan. Monitoring oxidative stress in patients with non-alcoholic and alcoholic liver diseases. Indian J Clin Biochem. 2005 July; 20(2): 24-28.
[18] Kasper DL, Fauci AS, Longo DL.,Braunwald E., Hauser SL. Jameson JL.Harrison's Priciples of Internal Medicine $16^{\text {th }}$ Edition.,2005,2:1813.

[19] Woolliams JA, Wiener G, Anderson PH, McMurray CH.Variation in the activities of glutathione peroxidase and superoxide dismutase and in the concentration of copper in the blood in various breed crosses of sheep. Res Vet Sci. (1983); 34(3): 253-6.

[20] Paglia, D.E. and Valentine, W.N. J. Studies on the quantitative and qualitative characterization of erythrocyte glutathione peroxidase. Lab. Clin. Med.( 1967); 70:158-69.

[21] Domninique General- Monnier, Irene Erdehneier,Jean Chandiere, Jean -Claude Yadan. Method of Colorimetric Analysis of Malondialdehyde and 4 Hydroxy -2-Enaldehydes as indices of lipid peroxidation: patent Number:5,726,063(1998).

[22] Aisen P.(1980) Iron transport and storage Proteins. Ann. Rev. Biochem, 49:357-393.

[23] Challand G.S. Mickaeldoudis A., Watfa R.R., Coles S.J., Macklin J.L.(1980), Distribution of haemoglobin in patients presenting to their general practitioner,and its correlation with serum ferritin. Ann. Clin. Biochem 27, 15-20.

[24] Kimber R.J., Rusaki Z., Blunden R.W.( 1983) Iron deficiency and iron overload: Serum ferritin and serum iron in clinical medicine. Pathology, 15:497-503.

[25] S.Bellentani,G Saccoccio, G costa, C Terribelli, F Manenti, M Sodde, L Saveria Croce’, F Sasso, G Pozzato, G Cristianini, G Brandi and the Dionysos study Group, Drinking habits as cofactors of risks for alcohol induced liver damage. (1997); Gut, 41:845-850.

[26] B Lavik, C Holmegaard, U Becker, Drinking patterns and biochemical signs of alcoholic liver disease in Danish and Greenlandic patients with alcohol addiction, 2006: Int J Circumpolar Health,:65(3):219-227.

[27] KrikunG., and Cederbaum A.I. Effect of chronic ethanol consumption on microsomal lipid peroxidation: Role of iron and comparision with controls.

[28] Harrison-Findik DD. Role of alcohol in the regulation of iron metabolism.World J. Gastroenterol.2007, 13(37):4925-4930.

[29] Clot P., Tabone M., Arico S., Albano E. Monitoring oxidative damage in patients with liver cirrhosis and different daily intake.Gut (1994);35:1637-1643.

[30] Bhandari S., Mukul P. Agarwal, S. Dwivedi, B.. Banerjee D., (2008). Monitoring oxidative stress across worsening child pugh class of cirrhosis. Indian J Med Sci, 62(11):444-451.

[31] Iqbal T.,Diad A., Ward DG., Brookes MJ., Tselepis C, Murray J, Elias E. (2009)Is Iron overload in alcohol-related cirrhosis mediated by Hepcidin? World J Gastroentrol, 15(46):5864-5866. 